Usán Supervía, P. \& Salavera Bordás, C. (2018). Orientaciones de logro, burnout académico y satisfacción intrínseca hacia la escuela en estudiantes de Educación Secundaria Obligatoria. Revista Electrónica Interuniversitaria de Formación del Profesorado, 21(3), 19-33.

DOI: http://dx.doi.org/10.6018/reifop.21.3.311371

\title{
Orientaciones de logro, burnout académico y satisfacción intrínseca hacia la escuela en estudiantes de Educación Secundaria Obligatoria
}

Pablo Usán Supervía, Carlos Salavera Bordás

Universidad de Zaragoza

\section{Resumen}

El objetivo del presente trabajo fue analizar la relación entre las orientaciones de logro, el burnout académico y la satisfacción intrínseca hacia la escuela en una muestra de 1756 alumnos $(M=14.47 ; \quad D T=1.63)$ pertenecientes a 12 centros de Educación Secundaria Obligatoria (ESO). Los instrumentos utilizados fueron las versiones españolas del Perception of Success Questionnaire (POSQ), el Maslach Burnout Inventory-Student Survey (MBI-SS) y la Intrinsic Satisfaction Classroom Scale (ISC). Los resultados mostraron relaciones significativas entre las orientaciones de logro hacia la tarea, eficacia académica y diversión en la escuela, en una línea de comportamientos más autodeterminados, así como la relación de las orientaciones de logro hacia el ego con el agotamiento físico/emocional, cinismo y aburrimiento, en otra línea de comportamientos menos autodeterminados. Se desprende la importancia de la promoción de comportamientos adaptativos orientados al esfuerzo, autoeficacia y diversión que mejoren el bienestar y vida académica de los estudiantes en sus centros educativos.

\section{Palabras clave}

Orientaciones de logro; burnout; satisfacción intrínseca; estudiantes. 


\title{
Achievement orientation, academic burnout and intrinsic school satisfaction in secondary education students
}

\begin{abstract}
The aim of the study was to analyze the relationship between burnout, engagement and academic self-efficacy in a sample of 1756 students $(M=14.47 ; S D=1.63)$ belonging to 12 secondary education high schools. The instruments used were the Spanish version of the Perception of Success Questionnaire (POSQ), the Maslach Burnout Inventory-Student Survey (MBI-SS), and the Intrinsic Satisfaction Classroom Scale (ISC) (Duda and Nicholls, 2002). The results showed significant relationships between task orientations, academic efficacy and fun at school, in a more self-determined behavior than the relationship of ego orientations with physical/emotional exhaustion, cynicism and boredom, in a less selfdetermined behavior. It is revealed the importance of promoting adaptive behaviors oriented to the effort, self-efficacy and fun that improve the well-being and academic life of the students in their high schools.
\end{abstract}

\section{Key words}

Achievement orientations; burnout; satisfaction; students.

\section{Introducción}

La investigación en educación ha sido y sigue siendo un foco de atención importante en nuestros días (Baena y Granero, 2015). En el proceso educativo de los estudiantes en los centros escolares se producen cuantiosas situaciones contextuales, situacionales y personales que pueden afectar de manera significativa al alumnado en su proceso de formación, más si cabe en enseñanza secundaria obligatoria y coincidiendo con la adolescencia, etapa de vital importancia en la vida de una persona y previo paso a la edad adulta (Gómez-Fraguela, Fernández, Romero y Luengo, 2008).

De esta manera, algunos estudiantes, en momentos más o menos transitorios en el tiempo, pueden no disponer o hacer uso de las estrategias y competencias necesarias para las exigencias y demandas de la vida académica en la que se hallan inmersos pudiendo llegar a experimentar sensaciones y percepciones que vayan en detrimento de su motivación y adhesión al estudio (Pino, Gutiérrez y Alvariñas, 2013; Usán, Salavera, Murillo y Mejías, 2016), padecimiento de agotamiento físico y psíquico (Palacio, Caballero, González, Gravini, 2012) o, incluso, la asunción de una actitud negativa e insatisfactoria de pérdida de interés hacia la propia institución escolar (Danielsen, Samdal, Hetland y Wold, 2009) que pueden conducir al fracaso escolar o abandono prematuro de sus estudios (Inglés, Martínez, García, Torregrosa y Ruiz, 2012).

Una de las teorías cognitivo-sociales más importantes en el estudio de la motivación escolar es la teoría de orientación al logro (Nicholls, 1989) la cual supone un marco de referencia muy utilizado en psicología de la educación (Ames, 1992). Ésta se refiere a los propósitos o razones que siguen los estudiantes para guiar su comportamiento en situaciones académicas. Así, en los entornos de logro, como la escuela, el objetivo fundamental del alumno es demostrar competencia o capacidad sugiriendo dos estados de implicación 
motivacional: uno, más autodeterminado, orientado hacia la tarea y, otro, menos autodeterminado, orientado hacia el ego.

De este modo, los alumnos orientados motivacionalmente hacia la tarea suelen poseer la creencia de que el éxito en la escuela proviene del esfuerzo, motivación y cooperación en el desarrollo de cualquier actividad; por otro lado, los orientados motivacionalmente hacia el ego, se relacionan con la posesión de habilidad o competencia superior respecto al resto de compañeros (Guivernau y Duda, 1994).

Numerosos estudios han relacionado positivamente las orientaciones motivacionales hacia la tarea con diversas variables como una mayor persistencia, voluntad y compromiso en la actividad escolar (Lukwu y Luján, 2011), motivación intrínseca en el desarrollo de las tareas (Casas, Baltatescu, Bertran, González y Hatos, 2013), mayor uso y desarrollo de las estrategias de afrontamiento (Salavera y Usán, 2017), esfuerzo y constancia (Lopez, 2008), diversión en el aula (Chu y Wang, 2012) así como un mayor bienestar físico, psicológico y emocional en la vida académica de los estudiantes (Gaeta, Cavazos, Sánchez, Rosario y Högemann, 2015).

Del mismo modo, unas orientaciones motivacionales hacia el ego se han relacionado con motivaciones extrínsecas en el desarrollo de actividades escolares (Vasters y Pillon, 2011), utilización de técnicas engañosas (DeFreese y Smith, 2013), falta de compromiso en el desarrollo de la actividad y abandono escolar (Downey, Rosengren y Donovan, 2011), problemas de ansiedad (Sebire, Standage y Vansteenkiste, 2009) así como, en definitiva, un menor bienestar psíquico y emocional del alumnado (Gillet, Vallerand y Paty, 2013).

En consecuencia, la teoría de la orientación al logro propone la adopción de una orientación motivacional hacia la tarea o el ego en función de cómo el alumno interpreta, responde y vive una actividad de logro con las implicaciones inherentes a cada una de ellas (Ames, 2002).

Por otro lado, desde un patrón de conducta más desadaptativo, ciertos alumnos durante su escolaridad pueden tener una pérdida de interés en sus estudios así como la aparición de dudas y/o contradicciones acerca de la propia capacidad personal en sacarlos adelante que, como ya hemos comentado, pueden desembocar en una desmotivación absoluta por parte de ciertos alumnos y posterior abandono de sus estudios (De Andrade, 2007; Musitu, Jiménez y Burgui, 2012). La presencia simultánea de estas manifestaciones se conoce como síndrome de burnout académico (Schaufeli, Martínez, Marques Pinto, Salanova y Bakker, 2002; Schaufeli y Salanova, 2007) caracterizado por tres dimensiones principales: cansancio emocional, cinismo y eficacia. El cansancio emocional se relaciona con el agotamiento físico y desgaste emocional que puede llegar a tener un alumno en un momento dado más o menos persistente durante su etapa escolar; el cinismo, hace referencia a la indiferencia o ausencia de interés hacia los estudios y, por último; la eficacia académica, se relaciona con la aptitud del alumno para el desempeño de sus tareas y quehaceres escolares.

De este modo, el burnout académico se ha relacionado con un bajo rendimiento escolar (Salanova, Martínez y Llorens, 2014), altos niveles de estrés (Extremera, Durán, y Rey, 2007; Salavera y Usán, 2017), bajos niveles de autoeficacia (Durán, Extremera, Rey, FernándezBerrocal, y Montalbán, 2006) así como escasa felicidad académica durante la etapa escolar del alumno (Salanova, Martínez, Bresó, Llorens y Grau, 2005).

Con todo esto, ciertas investigaciones han relacionado la orientación de logro de los estudiantes con sus niveles de burnout poniendo de manifiesto la existencia de una relación entre la orientación motivacional hacia la tarea y la eficacia académica en un patrón de conducta más autodeterminado (Salanova, Schaufeli, Martínez y Bresó, 2009; Bresó, 
Schaufeli y Salanova, 2011; Rodriguez-García, Sola-Martínez y Fernandez-Cruz, 2017) así como relaciones entre una orientación al logro hacia el ego con las dimensiones del cansancio emocional y cinismo, que caracterizan al burnout académico, en un patrón de conducta menos autodeterminado (Salanova, Del Líbano, Llorens y Schaufeli, 2014).

Por último, otro aspecto importante y ligado al devenir del alumno en su etapa escolar resulta la satisfacción intrínseca hacia la escuela. Desde la teoría del bienestar subjetivo (Diener, 2009) dicha satisfacción estaría compuesta por los componentes cognitivo (satisfacción con la vida) y afectivo (balance afectivo). Teniendo en cuenta estos dos componentes, la satisfacción con la vida se relacionaría como un compendio de las vivencias y experiencias propias de los estudiantes siendo, concretamente, el balance afectivo, el resultado de las reacciones inmediatas y continuas a los eventos que a ellos le suceden (Rodríguez y Goñi, 2011).

Por ende, la satisfacción intrínseca hacia la escuela es entendida como la evaluación cognitivo-afectiva que presentan los estudiantes con la propia experiencia escolar (Baena y Granero, 2015). Sin duda, el bienestar de los jóvenes en la escuela como institución tiene un gran peso en el crecimiento personal y académico del alumno (Fernández, 2011).

Según diferentes estudios, la satisfacción hacia la escuela resulta una variable determinante en el desarrollo del alumnado ya que, uno de los objetivos de la enseñanza es que, a la vez que el alumno aprenda, se divierta y esté satisfecho con los conocimientos que adquiere (Baena y Granero, 2015). Pero, además, la satisfacción adquiere connotaciones determinantes en el proceso de enseñanza-aprendizaje; por ejemplo, se ha podido comprobar que la satisfacción que los estudiantes sienten hacia la escuela se relaciona con los niveles de satisfacción general de su vida (Huebner, Gilman, Reschly y Hall, 2009), la disminución del estrés (Hui y Sun, 2010), mejora de su autoconcepto (Usán, Salavera, Murillo y Merino, 2018), relaciones sociales entre iguales (Skinner, Furrer, Marchand y Kinderman, 2008), compromiso hacia la escuela (Danielsen, Breivik, y Wold, 2011), motivaciones intrínsecas en el desarrollo de tareas (Casas, et al., 2013) así comola mejora del rendimiento académico de los alumnos (Salanova et al., 2014).

Por otro lado, la insatisfacción del alumnado hacia la escuela, se ha asociado con unos comportamientos menos autodeterminados como la disminución de la autoestima (Thøgersen-Ntoumani y Ntoumanis, 2007), el esfuerzo y la dedicación en la tarea académica (Reyes, 2009), diversión en la escuela (Chu y Wang, 2012) altos niveles de estrés (Tsouloupas, Carson, Matthews, Grawitch, y Barber, 2010), menor compromiso escolar (Busse y Walter, 2013) e, incluso, altas prevalencias de consumo de drogas en la adolescencia (Usán et al., 2016) que pueden conllevar a una disminución del bienestar físico, psicológico y emocional (Sebire, Standage y Vansteenkiste, 2009).

Por ende, se desprende la importancia que pueden tener las variables de orientación al logro, burnout académico y satisfacción intrínseca hacia la escuela, anteriormente enunciadas, en el desarrollo personal y académico del alumno en el transcurso de su vida escolar, las cuales, junto con otras variables contextuales, determinarán el devenir de los estudiantes en el sistema educativo en una antesala de su futuro personal y profesional más inmediato.

Por todo ello, y siguiendo a Moloi (2010), resultan necesarios más trabajos que indaguen en el estudio de la interrelación entre diferentes constructos psicológicos en la vida académica de los estudiantes a fin de mejorar su bienestar en aras de su crecimiento personal y académico dentro de la institución escolar. 
De esta manera, y ante los escasos estudios que relacionen de manera intrínseca las variables enunciadas, el principal objetivo del estudio se centra en analizar la relación entre la orientación al logro, el burnout académico y la satisfacción intrínseca hacia la escuela en una muestra de adolescentes escolares en la etapa de Educación Secundaria Obligatoria (ESO).

De esta manera, y en base al objetivo anteriormente enunciado, se postulan dos hipótesis: (a) Los estudiantes que posean una orientación de logro hacia la tarea se relacionarán positivamente con la eficacia académica y la diversión en la escuela en una línea de conductas más adaptativas que, (b) Aquellos estudiantes que posean una mayor orientación de logro hacia el ego, relacionados con un mayor agotamiento físico/emocional y cinismo unido del aburrimiento hacia la escuela, en otra línea de conductas más desadaptativas.

\section{Método}

\section{Diseño}

El presente trabajo, de acuerdo con Ato, López y Benavente (2013), consistió en un estudio ex post-facto de carácter prospectivo con un diseño descriptivo simple, respondiendo a un muestreo aleatorio simple.

\section{Muestra}

El estudio estuvo compuesto por 1756 estudiantes de género masculino ( $N=914 ; 52.05 \%$ ) y femenino ( $\mathrm{N}=842 ; 47.94 \%)$ pertenecientes a 12 centros públicos de Educación Secundaria Obligatoria (ESO) de la provincia de Zaragoza con edades comprendidas entre los 12 y 18 años $(M=14.47$; $D T=1.63)$.

Tabla 1. Edad y curso académico de los estudiantes.

\begin{tabular}{cccc}
\cline { 3 - 4 } & & $\mathbf{N}$ & $\%$ \\
\hline Edad & 12 años & 307 & 17.48 \\
& 13 años & 293 & 16.68 \\
& 14 años & 403 & 22.94 \\
& 15 años & 417 & 23.74 \\
& 16 años & 269 & 15.31 \\
& 17 años & 56 & 3.18 \\
& 18 años & 11 & 0.62 \\
\hline Curso académico & $1^{\circ}$ ESO & 338 & 19.24 \\
& $2^{\circ}$ ESO & 436 & 24.82 \\
& $3^{\circ}$ ESO & 567 & 32.28 \\
& $4^{\circ}$ ESO & 415 & 23.63 \\
\hline
\end{tabular}




\section{Instrumentos de evaluación}

Para recoger la información y aproximarnos al objetivo establecido se utilizaron tres cuestionarios.

Con la finalidad de aproximarnos a las orientaciones de logro de los alumnos se utilizó el Perception of Success Questionnaire (POSQ) de Roberts, Treasure y Balagué (1998) traducida y validada al castellano por Martínez, Alonso y Moreno (2006). El cuestionario se compone de 12 ítems que miden las orientaciones de logro mediante dos dimensiones; orientación hacia la tarea (6) $(\alpha=.85)$ ( $\mathrm{Ej}$. "En las clases siento que tengo éxito cuando realizo las tareas lo mejor que puedo") y orientación hacia el ego (6) ( $\alpha=.82)$ (Ej. "En las clases siento que tengo éxito cuando demuestro al profesor y mis compañeros que soy el mejor"). Las respuestas responden al grado de acuerdo o desacuerdo con los ítems en una escala tipo Likert que oscila desde "Muy en desacuerdo" (1) hasta "Muy de acuerdo" (5). La fiabilidad del cuestionario ha sido demostrada en diferentes estudios en el contexto escolar obteniendo un alpha de Cronbach de .85 para la subescala tarea y de .82 para la subescala ego (Treasure y Roberts, 1994) siendo de .84 y .85 respectivamente, en nuestro estudio.

Con la finalidad de percibir el burnout académico de los estudiantes se utilizó la versión española del Maslach Burnout Inventory - Student Survey (MBI-SS) (Schaufeli et al., 2002). Dicha cuestionario se compone de 15 ítems repartidos en tres dimensiones: agotamiento físico/emocional (5) ( $\alpha=.81$ ) (Ej. "Estudiar o ir a clase todo el día es una tensión para mí); cinismo (4) ( $\alpha=$.79) (Ej. "He perdido entusiasmo por mis estudios") y eficacia (6) ( $\alpha=.77$ ) (Ej. "Me estimula conseguir objetivos en mis estudios"). Las respuestas oscilan en una escala tipo Likert desde "Muy en desacuerdo" (1) hasta "Muy de acuerdo" (5). El instrumento original denota un alpha de Cronbach de .79 mientras que en nuestra investigación otorgó una prevalencia de .81 .

Para averiguar la satisfacción intrínseca de los discentes se partió de la versión española de Intrinsic Satisfaction Classroom Scale (ISC) (Duda y Nicholls, 2002). El instrumento está compuesto por 7 ítems los cuales se dividen en dos dimensiones: diversión (5) ( $\alpha=.77)$ (Ej. "Disfruto aprendiendo en el colegio") y aburrimiento (2) ( $\alpha=.76)$ (Ej. "Deseo que se pase rápido el tiempo en el colegio"). Las respuestas se erigen en una escala tipo Likert desde "Muy en desacuerdo" (1) hasta "Muy de acuerdo" (5). La fiabilidad del instrumento original denota un alpha de Cronbach de .77 mientras que en nuestra investigación otorgó una prevalencia de .79 .

\section{Procedimiento}

En la realización del estudio se contó con la aprobación de los diferentes centros de educación secundaria así como de los padres/tutores de los alumnos por medio de consentimiento informado para poder participar en la investigación. Un día de la semana, acordado previamente con jefatura de estudios, se llevó a cabo la cumplimentación de los cuestionarios en cada una de las clases de un mismo centro. Todos los sujetos y sus padres/tutores fueron previamente informados de la naturaleza del estudio participando de manera voluntaria y respetando, de este modo, las directrices éticas de la Declaración de Helsinki (AMM, 2000) en todos sus términos.

\section{Análisis de datos}

Se procedió a realizar una estadística descriptiva con la finalidad de conocer los datos sociodemográficos de la muestra así como las diferentes variadas estudiadas. Posteriormente, se llevaron a cabo correlaciones entre las variables de orientaciones al logro, burnout académico y satisfacción intrínseca hacia la escuela, procesadas y analizadas mediante el programa estadístico IBM SPSS v22.0. Finalmente, se planteó un modelo de 
ecuaciones estructurales, mediante el método de extracción de máxima verosimilitud, que permitiese validar y cuantificar las relaciones causales entre el burnout, engagement y autoeficacia mediante el programa AMOS v24. Para todas las operaciones, se tuvo en cuenta un nivel de significación $\mathrm{p} \leq 0,05$ trabajándose con un nivel de confianza del 95\%.

\section{Resultados}

A continuación se presentan los resultados obtenidos en las diferentes variables estudiadas:

\section{Variables descriptivas}

Tal y como se refleja en la tabla 2, las puntuaciones de las diferentes dimensiones resultan heterogéneas. La variable de orientación motivacional hacia la tarea $(M=3,80)$ obtuvo mayor puntuación que el resto de dimensiones que componen el burnout y la satisfacción intrínseca hacia la escuela. En el primero, destaca la eficacia académica $(M=3,30)$ y el agotamiento físico/emocional $(M=3,19)$ por encima del cinismo $(M=2,14)$. Respecto a la segunda, destaca la dimensión de aburrimiento $(M=3,43)$ por delante de la diversión $(M=2,84)$.

Tabla 2. Descriptivos de las variables de orientación de logro, burnout académico y satisfacción intrínseca hacia la escuela

\begin{tabular}{cccc}
\cline { 2 - 4 } & Media & DT & $\begin{array}{c}\text { Alfa de } \\
\text { Cronbach }\end{array}$ \\
\hline Orientación de logro Tarea & 3.80 & .80 & .85 \\
Ego & 2.89 & 1.03 & .82 \\
\hline Burnout académico Agotamiento físico/emocional & 3.19 & .96 & .81 \\
Cinismo & 2.14 & 1.06 & .79 \\
Eficacia & 3.30 & .78 & .77 \\
\hline Satisfacción intrínseca Diversión & 2.84 & .90 & .78 \\
Aburrimiento & 3.43 & 1.04 & .76 \\
\hline
\end{tabular}

Relación entre las variables de orientación de logro, burnout académico y satisfacción intrínseca hacia la escuela

Analizando las variables de orientación de logro, burnout y satisfacción intrínseca hacia la escuela se apreciaron correlaciones significativas (véase Tabla 3).

Por un lado, entre las dimensiones de la orientación de logro, se aprecia la fuerte relación de la orientada hacia la tarea con la eficacia académica del burnout ( $r=.520)$ así como la diversión, de la satisfacción intrínseca hacia la escuela $(r=.429)$. Al mismo tiempo, se denotan relaciones significativas inversas de la misma con el agotamiento físico/emocional $(r=.-182)$, el cinismo ( $r=.-434)$ y el aburrimiento $(r=-.102)$. Por otro lado, la orientación de logro hacia el ego se vincula significativamente con el agotamiento físico/emocional ( $r=.132)$ y el cinismo ( $r=.164)$ del burnout, así como el aburrimiento $(r=.166)$ de la satisfacción intrínseca hacia la escuela. 
En referencia a las dimensiones del burnout, el agotamiento físico/emocional y el cinismo correlacionan entre sí $(r=.328)$ además de hacerlo positivamente con el aburrimiento y, de manera inversa, con la diversión.

Por último, en la satisfacción intrínseca hacia la escuela, diversión y aburrimiento correlacionan entre sí de manera inversa $(r=-325)$.

Tabla 3. Relación entre variables de orientaciones de logro, burnout académico y satisfacción intrínseca hacia la escuela.

\begin{tabular}{llllll}
\hline 1 & 2 & 3 & 4 & 5 & 6 \\
\hline
\end{tabular}

\begin{tabular}{|c|c|c|c|c|c|c|c|}
\hline \multicolumn{2}{|c|}{ Orientación de logro 1. Tarea } & \multicolumn{2}{|l|}{1} & & & & \\
\hline & 2. Ego & $.289 * *$ & 1 & & & & \\
\hline \multirow[t]{3}{*}{ Burnout académico 3} & $\begin{array}{l}\text { 3. Agotamiento } \\
\text { físico/emocional }\end{array}$ & $-.182 * *$ & $.132 * *$ & 1 & & & \\
\hline & 4. Cinismo & $-.434 * *$ & $.164 * *$ & $.328 * *$ & 1 & & \\
\hline & $\begin{array}{l}\text { 5. Eficacia } \\
\text { profesional }\end{array}$ & $.520 * *$ & $.256 * *$ & $-.158 * *$ & $-.489 * *$ & 1 & \\
\hline \multicolumn{2}{|c|}{ Satisfacción intrínseca 6. Diversión } & $.429 * *$ & .042 & $-.307^{* *}$ & $-.296 * *$ & $.472 * *$ & 1 \\
\hline & 7. Aburrimiento & $-.102 * *$ & $.166 * *$ & $.286 * *$ & $.223 * *$ & $-.154^{* *}$ & $-.325 * *$ \\
\hline
\end{tabular}

** La correlación es significativa al nivel 0,01 (bilateral)

* La correlación es significante al nivel 0,05 (bilateral)

Modelo de ecuaciones estructurales entre las variables de orientación de logro, burnout académico y satisfacción intrínseca hacia la escuela

Por último, la figura 1 muestra el resultado del análisis con ecuaciones estructurales mediante el método de extracción de máxima verosimilitud, confirmando la adecuación del modelo compuesto por los constructos planteados en el estudio.

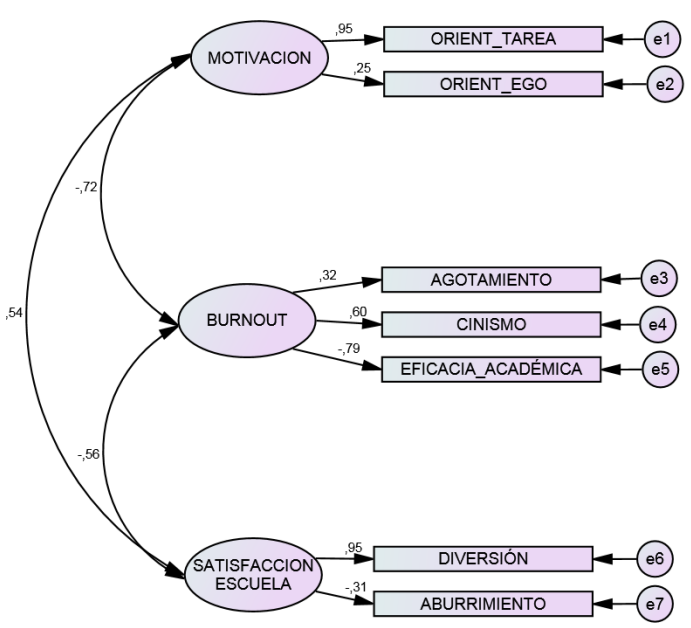

Figura 1. Modelo de ecuaciones estructurales entre las variables de orientación de logro, burnout académico y satisfacción intrínseca hacia la escuela 
El modelo refleja una alta correlación entre las orientación de logro y el burnout $(r=-.76)$ de la misma manera que con la satisfacción intrínseca hacia la escuela $(r=-.66)$ lo que nos indicaría que altos niveles de burnout llevarían aparejados menores prevalencias de orientación de logro y satisfacción intrínseca hacia la escuela. Por otra parte, la orientación de logro y la satisfacción intrínseca hacia la escuela correlación entre sí $(r=.52)$ indicándonos la relación existente entre ellas. En cuanto al ajuste del modelo, los diversos índices resultaron adecuados, por lo que se puede afirmar que el modelo propuesto acerca de la estructura factorial entre las orientaciones de logro, el burnout académico y la satisfacción intrínseca hacia la escuela resulta sustentable: $\chi 2(14)=75.558, p<0.001 ; \chi 2 / g \mid=5.397 ; C F I=$ $0.94 ; \mathrm{NFI}=0.94 ; \mathrm{TLI}=0.85 ; \mathrm{RMSEA}=0.071, \mathrm{IC} 95 \%(0.056-0.087)$.

\section{Discusión}

El objetivo del estudio fue analizar la relación entre las orientaciones de logro, burnout académico y satisfacción intrínseca hacia la escuela en una muestra de estudiantes adolescentes de Educación Secundaria Obligatoria.

La primera hipótesis que se postuló fue, precisamente, que aquellos estudiantes con una mayor orientación de logro escolar hacia la tarea se relacionarían con la eficacia académica y la diversión hacia la escuela, en una línea de conductas más adaptativas. Dicha hipótesis se cumplió en su totalidad; los resultados de nuestra investigación revelaron la estrecha relación entre las orientaciones de logro hacia la tarea en la asunción, por parte de los estudiantes, de que el éxito en la escuela proviene del esfuerzo, interés y motivación con una mayor presunción de autoeficacia académica ligado a la satisfacción intrínseca de diversión hacia ella.

Por un lado, diversos estudios ponen de manifiesto la relación entre orientaciones de logro hacia la tarea, eficacia académica y diversión. Ferriz, Sicilia y Sáenz (2013) en una muestra de estudiantes en la etapa de Secundaria encuentran relaciones entre los alumnos más motivados intrínsecamente hacia la tarea y su percepción de eficacia académica en el desempeño de la actividad escolar. Además, denotan una estrecha vinculación entre la diversión y bienestar hacia la propia institución escolar. Cuevas, García y Contreras (2013) predicen la orientación motivacional hacia la tarea en los niveles de satisfacción y disfrute del alumnado. Bresó, Schaufeli y Salanova (2011) definen un patrón de conducta más autodeterminadas en el que la dimensión de eficacia académica del burnout se relaciona positivamente con orientaciones de logro orientadas hacia la tarea dejando de lado el aburrimiento y el agotamiento físico y emocional de los estudiantes en las clases.

Por otro lado, continuando con esta línea de conductas adaptativas, ciertas investigaciones relacionan las variables objeto de estudio con otras académicas y/o contextuales. De este modo, ciertos trabajos relacionan positivamente las orientaciones de logro hacia la tarea con una mayor dedicación, motivación y disfrute escolar (Casas, et al., 2013), utilización de estrategias de afrontamiento que conducen a una mayor eficacia académica (Salavera y Usán, 2017), mayor compromiso y adhesión con las tareas y demandas académicas (Skinner, Furrer, Marchand y Kinderman, 2008), mejora del rendimiento académico (Salanova et al., 2014) así como, en definitiva, un mayor bienestar psicológico y emocional (Gaeta, Cavazos, Sánchez, Rosario y Högemann (2015).

La segunda hipótesis de nuestro estudio aludía a la posible relación entre una orientación de logro hacia el ego con el agotamiento físico/emocional, cinismo y aburrimiento de los estudiantes, en una línea de conductas más desadaptativas. 
Dicha hipótesis se volvió a cumplir en su totalidad; los resultados de nuestra investigación arrojaron las relaciones entre unas orientaciones de logro hacia el ego con el agotamiento físico/emocional y el cinismo así como el aburrimiento en la escuela. Es decir, los estudiantes de nuestra investigación más orientados hacia la creencia de que el éxito en la escuela proviene de la posesión de habilidad o competencia superior respecto al resto se relacionaron con un mayor índice de agotamiento y cinismo teniendo, en consecuencia, un considerable aburrimiento hacia la institución escolar.

Dichos resultados son corroborados por algunos estudios de la literatura científica. Baena, Granero, Pérez, Bracho y Sánchez (2013) exponen que los estudiantes que perciben mayor agotamiento experimentan mayor cinismo y, por tanto, menor autoeficacia, asociándose negativamente con la satisfacción en sus estudios así como en orientaciones de logro más extrínsecos. Méndez, Fernández, Cecchini y González (2012) reflejan que las orientaciones de logro hacia el ego predicen el aburrimiento en la escuela unido a otros factores desadaptativos como el cansancio, menor persistencia en el aprendizaje o incluso la cohesión de grupo. Salanova et al., (2014) mantienen la relación inequívoca de las orientaciones de logro hacia el ego con las dimensiones del cansancio emocional y cinismo, que caracterizan al burnout académico. Además, las orientaciones de logro hacia el ego se relacionan con un bajo rendimiento escolar (Salanova, Martínez y Llorens, 2014) que puede conducir a aumentar los niveles de estrés y fatiga (Tsouloupas, Carson, Matthews, Grawitch, y Barber, 2010) pudiendo, incluso, estar vinculadas con el inicio o mantenimiento del consumo de drogas en adolescentes (Usán, 2014).

Del mismo modo, unas orientaciones motivacionales hacia el ego se han relacionado con motivaciones extrínsecas y alejadas de comportamientos adaptativos en el desarrollo de actividades escolares (DeFreese y Smith, 2013) conduciendo a un menor compromiso y dedicación en ellas (Downey, Rosengren y Donovan, 2011) así como un menor bienestar psíquico y emocional del alumnado (Gillet, Vallerand y Paty, 2013) pudiendo ser un factor desencadenante del abandono y/o fracaso escolar por parte de los estudiantes (Bresó, Schaufeli y Salanova, 2011; Musitu, Jiménez y Murgui, 2013).

Por todo esto, tal y como venimos comentando, se aprecia la importancia de las orientaciones de logro de los estudiantes que, unidas a sus niveles de burnout académico y satisfacción intrínseca hacia la escuela, afectan al devenir del alumno en el proceso educativo pudiendo condicionar su rendimiento y adherencia hacia la propia institución escolar (Ervasti et al., 2011) resultando fundamental atender a ellos para un óptimo desarrollo de su vida académica (Bresó, Schaufeli y Salanova, 2011; Salanova et al., 2014; Vera, Le Blanc, Taris y Salanova, 2014).

\section{Limitaciones del estudio}

Las limitaciones del estudio pueden residir en el diseño transversal del mismo tomando los datos en un momento espacio temporal dado. A su vez, los centros de educación secundaria encuestados responden de una manera aleatoria a su inclusión en el estudio no tomando una muestra uniforme de todos los barrios o zonas de la ciudad pudiendo afectar, en consecuencia, a las posibles diferencias entre sus niveles socioeconómicos o educativos así como a otras cuestiones sociales. A la par, las prevalencias de orientación de logro, burnout y satisfacción intrínseca hacia la escuela pueden variar de un curso escolar a otro, e incluso dentro de un mismo año, dependiendo de las circunstancias personales y contextuales de los estudiantes en su grupo/clase. 


\section{Prospectivas de futuro}

Como prospectivas de futuro resultarían interesantes modelos longitudinales que permitiesen valorar la evolución de los constructos estudiados a lo largo de un período más largo de tiempo. Además, sería importante atender otras etapas educativas como pueden ser Educación Primaria o Bachillerato, de la misma manera que otras etapas de la vida académica del estudiante como pueden ser los Grados de Formación Medio o Superior así como la propia etapa universitaria. Del mismo modo, resultaría relevante atender a otras variables, en relación con las estudiadas, como pueden ser el género, la tipología de colegio o el propio rendimiento escolar de los alumnos así como aspectos sociales y culturales, entre otros.

\section{Implicaciones prácticas}

Este trabajo puede tener unas implicaciones de carácter práctico que desemboquen en unas estrategias didácticas a utilizar sobre los alumnos a través de los propios docentes u orientadores desde edades tempranas orientadas hacia conductas autodeterminadas como la promoción del esfuerzo, constancia y/o diversión en el estudio que hagan a los alumnos sentirse eficaces en el desempeño de sus tareas académicas en aras de una mayor persistencia y dedicación que puedan servir, a la par, como factor de protección del bajo rendimiento académico y adecuada vida académica.

A su vez, programas de intervención dirigidos por profesionales del ámbito en la apuesta por parte de la Administración y/o los propios centros educativos por el trabajo con sus adolescentes en las variables acaecidas puede ayudar a contribuir a la formación integral y educacional del alumno disminuyendo el prematuro abandono escolar e incluso potenciando su rendimiento académico.

Por último, los resultados de nuestra investigación animan a seguir investigando y buscar nuevas preguntas que nos ayuden a definir metodologías encontrando respuestas que nos permitan avanzar en la construcción del desarrollo de los adolescentes escolares.

\section{Referencias}

Ames, C. (1992). Achievement goals, motivational climate and motivational processes. En G.C. Roberts (Ed.) Motivation in sport and exercise (pp. 161-176). Champaign, IL: Human Kinetics.

Asociación Médica Mundial (AMM) (2000). Declaración de Helsinki. Principios éticos para las investigaciones con los seres humanos. Seúl (Corea).

Ato, M., López, J. y Benavente, A. (2013). Un sistema de clasificación de los diseños de investigación en psicología. Anales de psicología, 29(3), 1038-1059.

Baena, A. y Granero, A. (2015). Modelo de predicción de la satisfacción con la educación física y la escuela. Revista de Psicodidáctica, 20(1), 177-192.

Baena, A., Granero, A., Sánchez, J. A. y Martínez, M. (2013). Apoyo a la autonomía en educación física: Antecedentes, diseño, metodología y análisis de la relación con la motivación en estudiantes adolescentes. Retos. Nuevas tendencias en Educación Física, Deportes y Recreación, 24, 46-49.

Bresó, E., Schaufeli, W. B. y Salanova, M. (2011). Can a self-efficacy-based intervention decrease burnout, increase engagement, and enhance performance? A quasiexperimental study. Higher Education, 61, 339-355. 
Busse, V. y Walter, C. (2013). "Foreign Language Learning Motivation in Higher Education: A Longitudinal Study of Motivational Changes and Their Causes", en The Modern Language Journal, 97(2), 435-456.

Casas, F., Baltatescu, S., Bertran, I., González, M. y Hatos, A. (2013). "School Satisfaction Among Adolescents: Testing Different Indicators for its Measurement and its Relationship with Overall Life Satisfaction and Subjective Well-Being in Romania and Spain", en Social Indicators Resesearch, 111, 665-681.

Chu, A. y Wang, C. (2012). Differences in level of sport commitment among college dance sport competitors. Social Behavior and Personality, 40(5), 755-766.

Cuevas, R., García, T. y Contreras, O. (2013). Perfiles motivacionales en educación física: Una aproximación desde la teoría de las Metas de Logro 2x2. Anales de Psicología, 29(3), 685-692. doi: 10.6018/analesps.29.3.175821.

Danielsen, A., Breivik, K. y Wold, B. (2011). Do Perceived Academic Competence and School Satisfaction Mediate the Relationships Between Perceived Support Provided by Teachers and Classmates, and Academic Initiative? Scandinavian Journal of Educational Research, 55(4), 379-401.

Danielsen, A., Samdal, O., Hetland, J. y Wold, B. (2009). School-related social support and students' perceived life satisfaction. Journal of Education Research, 102(4), 303-318.

De Andrade, T.M. (2007). Drug use among young adolescents - prospects for prevention within the context of family relationships and education in the light of damage control principles and practices. Ciencia e Saude Coletiva, 12(5), 1118-1120.

DeFreese, J. y Smith, A. (2013). Teammate social support, burnout, and self determinated motivation in collegiate athletes. Psychology of Sport and Exercise, 14(2), 258-265.

Diener, E. (2009). Assessing well-being: Progress and opportunities. En E. Diener (Ed.), Assessing well-being. The collected works of Ed Diener (pp. 25- 65). New York: Springer (Social Indicators Research Series, 39).

Downey, L., Rosengren, D. y Donovan, D. (2011). Sources of motivation for abstinence: A replication analysis of the reasons for quitting questionnaire. Addictive Behaviors, 26(1), 79-89.

Duda, J.L. y Nicholls, J. (1992). Dimensions of achievement motivation in schoolwork and sport. Journal of Educational Psychology, 84(3), 290-299.

Durán, A., Extremera, N., Rey, L., Fernández-Berrocal, P. y Montalbán, M. (2006). Predicting academic burnout and engagement in educational settings: Assessing the incremental validity of perceived emotional intelligence beyond perceived stress and general self-efficacy. Psicothema, 18(1), 158-164.

Ervasti, J., Kivima, M., Puusniekka, R., Luopa, P., Pentti, J., Suominen, S. y Virtanen, M. (2011). Students'school satisfaction as predictor ofteachers' sickness absence: A prospective cohort study. The European Journal of Public Health, 22(2), 215-219.

Extremera, N., Durán, A. y Rey, L. (2007). Inteligencia emocional y su relación con los niveles de burnout, engagement y estrés en estudiantes universitarios. Revista de Educación, 342(2), 239-256.

Fernández, E. (2011). "El valor del realismo en las tareas de educación física bilingüe”, en Revista Digital Educativa Wadired (1). 
Ferriz, R., Sicilia, A. y Sáenz, P. (2013). Predicting satisfaction in physical education classes: A study based on self-determination theory. The Open Education Journal, 6, 1-7.

Gaeta, M, Cavazos, J., Sánchez, A.P., Rosario, P. y Högemann, J. (2015). Propiedades psicométricas de la versión mexicana del Cuestionario para la Evaluación de Metas Académicas (CEMA). Revista Latinoamericana de Psicología, 47(1), 16-24.

Gillet, N., Vallerand, R. y Paty, B. (2013). Situational motivational profiles and performance with elite performers. Journal of Applied Social Psychology, 43(6), 1200-1210.

Gómez-Fraguela, J.A., Fernández, N., Romero, E. y Luengo, A. (2008). El botellón y el consumo de alcohol y otras drogas en la juventud. Trastornos Adictivos, 20(2), 211217.

Guivernau, M. y Duda, J. L. (1994). Psychometric properties of a spanish version of the task and ego orientation in sport questionnaire (TEOSQ) and beliefs about the causes of success inventory. Revista de Psicología del Deporte, 5, 31-51.

Huebner, E., Gilman, R., Reschly, A. y Hall, R. (2009). Positive schools. En S. J. Lopez (Ed.), Handbook of positive psychology (2nd ed.). New York: Plenum, 445-455.

Hui, E.K. y Sun, R.C. (2010). Chinese children's perceived school satisfaction: the role of contextual and intrapersonal factors. Educational Psychology: An International Journal of Experimental Educational Psychology, 30(2), 155--172.

Inglés, C., Martínez-González, A., García-Fernández, J.M., Torregrosa, M. y Ruiz Esteban, C. (2012). Prosocial behavior and self-concept of spanish students of compulsory secondary education. Revista de psicodidáctica, 17(1), 135-156.

López, M. (2008). Un modelo causal de las orientaciones de meta en el ámbito deportivo: deportes individuales y en equipo. Tesis Doctoral. Universidad de Alicante.

Lukwu, R.M. y Luján, J.F. (2011). Sport commitment and adherence: A social-cognitive analysis. Revista Internacional de Ciencias del Deporte, 7(25), 277-286.

Martínez, C., Alonso, N. y Moreno, J.A. (2006). Análisis factorial confirmatorio del "Cuestionario de Percepción de Éxito (POSQ)" en alumnos adolescentes de Educación Física. En M. A. González, J. A. Sánchez y A. Areces (Eds.), IV Congreso de la Asociación Española de Ciencias del Deporte (757-761). A Coruña: Xunta de Galicia.

Méndez, A., Fernández, J., Cecchini, J.A. y González, C. (2013). Perfiles motivacionales y sus consecuencias en educación física. Un estudio complementario de metas de logro $2 \times 2$ y autodeterminación. Revista de Psicología del Deporte, 22(1), 29-38.

Moloi, K. C. (2010). How can schools build learning organizations in difficult education contexts? South African Journal of Education, 30(4), 621-633.

Musitu, G. Jiménez, T. y Murgui, S. (2012). Funcionamiento familiar, autoestima y consumo de sustancias en adolescentes: un modelo de mediación. Revista de salud pública de México, 49(1), 3-10.

Nicholls, J.G. (1989). Achievement motivation: Conceptions of ability, subjective experience, task choice and performance. Psychological Review, 21, 328-346.

Palacio, S., Caballero, C., González, O., Gravini, M. y Contreras, K. (2012). Relación del burnout y las estrategias de afrontamiento con el rendimiento académico en estudiantes universitarios. Universytas Psychological, 11(2), 234-239.

Pino, M., Gutiérrez, A. y Alvariñas, M. (2013). Estudio sobre los hábitos de actividad física saludable en futuros docentes. Journal Of Human Sport and Exercise, 8(2), 210-216. 
Reyes, M. (2009). Clima motivacional y orientaciones de meta en futbolistas peruanos de primera división. Cuadernos de Psicología del Deporte, 9(1), 5-20.

Roberts, G., Treasure, D. y Balagué, G. (1998). Achievement goals in sport: The development and validation of the Perception of Success Questionnaire. Journal of Sport Sciences, $16,337-347$.

Rodríguez, A., y Goñi, A. (2011). La estructura tridimensional del bienestar subjetivo. Anales de Psicología, 27(2), 327-332.

Rodriguez-García, A.M., Sola-Martínez, T. y Fernandez-Cruz, M. (2017). Impacto del burnout en el desarrollo profesional del profesorado universitario. Una revisión de la investigación. Revista universitaria de Formación del Profesorado, 20(3). 161-178. DOI: http://dx.doi.org/10.6018/reifop.20.3.275121

Salanova, M., Del Líbano, M., Llorens, S. y Schaufeli, W. (2014). Engaged, workaholic, burned-out or just 9-to-5? Toward a typology of employee well-being. Stress and Health, 30, 71-81.

Salanova, M., Martínez, I.M., y Llorens, S. (2014). Una mirada más" positiva" a la salud ocupacional desde la Psicología Organizacional Positiva en tiempos de crisis: aportaciones desde el equipo de investigación WoNT. Papeles del psicólogo, 35, 2230.

Salanova, M., Martínez, I., Bresó, E., Llorens, S., y Grau, R. (2005). Bienestar psicológico en estudiantes universitarios: facilitadores y obstaculizadores del desempeño académico. Anales de Psicología, 21, 170-180.

Salanova, M., Schaufeli, W., Martínez, I. y Bresó, E. (2009). How obstacles and facilitators predict academic performance: The mediating role of study burnout and engagement. Anxiety, Stress and Coping, 23, 53-70.

Salavera, C. y Usán, P. (2017). Relación entre los estilos de humor y la satisfacción con la vida en estudiantes de Secundaria. European Journal of Investigation in Health, Psychology and Education, 7(2), 87-97.

Schaufeli, W.B., Martínez, I., Marques-Pinto, A., Salanova, M. y Bakker, A. (2002). Burnout and engagement in university students: A crossnational study. Journal of CrossCultural Psychology, 33(5), 464-468.

Schauffeli, W.B. y Salanova, M. (2007). Efficacy or inefficacy, that's the question: Burnout and work engagement, and their relationships with efficacy believes. Anxiety, Stress and Coping, 20, 177-196.

Sebire, S., Standage, M. y Vansteenkiste, M. (2009). Examining intrinsic versus extrinsic exercise goals: Cognitive, affective, and behavioral outcomes. Journal of Sport and Exercise Psychology, 31, 189-210.

Skinner, E., Furrer, C., Marchand, G. y Kinderman, T. (2008). Engagement and disaffection in the classroom: Part of a larger motivational dynamic? Journal of Educational Psychology, 100, 765-781.

Thøgersen, C. y Ntoumanis, N. (2007). A self-determination theory approach to the study of body image concerns, self-presentation and selfperceptions in a sample of aerobic instructors. Journal of Health Psychology, 12, 301-315.

Tsouloupas C., Carson, R., Matthews, R., Grawitch, M. y Barber, L. (2010). Exploring the association between teachers' perceived student misbehaviour and emotional 
exhaustion: The importance of teacher efficacy beliefs and emotion regulation. Educational Psychology, 30, 173-189.

Usán, P. (2014). Consumo de drogas y variables psicológicas deportivas en futbolistas adolescentes. Tesis Doctoral. Universidad de Zaragoza.

Usán, P., Salavera, C., Murillo, V. y Mejías J.J. (2016). Relación entre motivación, compromiso y autoconcepto físico en futbolistas adolescentes de diferentes categorías deportivas. Cuadernos de Psicología del Deporte, 16(1), 183-194.

Vasters, G. y Pillon, S. (2011). Drugs use by adolescents and their perceptions about specialized treatment adherence and dropout. Revista Latino Americana de Enfermagem, 19(2), 317-324.

Vera, M., Le Blanc, P., Taris, T. y Salanova, M. (2014). Patterns of engagement: the relationship between efficacy beliefs and task engagement at the individual versus collective level. Journal of Applied Social Psychology, 44, 133-144. 